\title{
Capacidade aquisitiva e disponibilidade de medicamentos para doenças crônicas no setor público
}

\author{
Ana Paula Helfer, ${ }^{1}$ Aline Lins Camargo, ${ }^{2}$ Noemia Urruth Leão Tavares, ${ }^{3}$ \\ Panos Kanavos ${ }^{4}$ e Andréa Dâmaso Bertoldi 5
}

Como citar Helfer AP, Camargo AL, Tavares NUL, Kanavos P, Bertoldi AD. Capacidade aquisitiva e disponibilidade de medicamentos para doenças crônicas no setor público. Rev Panam Salud Publica. 2012:31(3):225-32.

RESUMO Objetivo. Avaliar a capacidade aquisitiva do trabalhador para pagar medicamentos utilizados no tratamento de doenças crônicas e a disponibilidade desses medicamentos na forma de referência, similar ou genérica para fornecimento gratuito no setor público.

Métodos. Utilizou-se metodologia preconizada pela Organização Mundial da Saúde (OMS) $e$ Health Action International (HAI) para coleta padronizada de informações sobre preços de venda no setor privado e disponibilidade no setor público de medicamentos em seis cidades do Rio Grande do Sul, Brasil. A coleta de dados ocorreu de novembro de 2008 a janeiro de 2009. A capacidade aquisitiva foi estimada como o número de dias do salário que um trabalhador com rendimento de 1 salário mínimo nacional necessita trabalhar para adquirir, em uma farmácia privada, a quantidade necessária de medicamento para 1 mês de tratamento. A disponibilidade foi avaliada verificando-se a presença dos medicamentos nas farmácias do setor público.

Resultados. A pesquisa incluiu 22 estabelecimentos públicos e 30 farmácias privadas. Dos 21 medicamentos utilizados no tratamento de sete doenças crônicas, apenas nove eram disponibilizados gratuitamente nos seis municípios pesquisados. O percentual médio da disponibilidade variou de 83,3\% (São Leopoldo) a 97,6\% (Caxias do Sul). A capacidade aquisitiva variou de 0,4 a 10,5 dias de salário para medicamentos de referência; de 0,2 a 8,4 dias de salário para medicamentos similares; e de 0,3 a 3,8 dias de salário para medicamentos genéricos.

Conclusões. A disponibilidade geral dos medicamentos pesquisados foi superior aos $80 \%$ recomendados pela OMS; porém, alguns tratamentos não estavam disponíveis, ou apresentaram uma disponibilidade limitada no setor público. A capacidade aquisitiva dos trabalhadores nos municípios estudados indicou um comprometimento de dias do salário que pode afetar a continuidade dos tratamentos com medicamentos para doenças crônicas.

Palavras-chave Economia farmacêutica; medicamentos essenciais; preço de medicamento; doença crônica.

1 Universidade do Vale do Rio dos Sinos (UNISINOS), Programa de Pós-Graduação em Saúde Coletiva, São Leopoldo (RS), Brasil. Correspondência: aninhahelfer@yahoo.com.br

2 Universidade Federal de Ciências da Saúde de Porto Alegre, Departamento de Ciências Básicas da Saúde, Porto Alegre (RS), Brasil.

3 Universidade da Região da Campanha, Centro de Ciências da Saúde, Bagé (RS), Brasil.
Com as mudanças decorrentes da transição epidemiológica e demográfica, tornaram-se predominantes as doenças

\footnotetext{
4 The London School of Economics and Political Science, London, United Kingdom.

5 Universidade Federal de Pelotas, Centro de Pesquisas Epidemiológicas, Pelotas (RS), Brasil.
}

crônicas e suas complicações, que, muitas vezes, implicam anos de utilização dos serviços de saúde. Com isso, aumentam os gastos (1) e a demanda por medicamentos, especialmente os de uso contínuo (2).

Os gastos com medicamentos contribuem significativamente para o gasto total com saúde e têm aumentado ao longo 
do tempo, considerando tanto os gastos da população quanto do governo. No Brasil, os medicamentos representavam $37 \%$ dos gastos familiares com saúde em 1995 e 1996 (3), passando para 40,6\% em 2002 e 2003 (4) e para 47\% em 2008 (5). $\mathrm{O}$ gasto do governo com medicamentos para fornecimento gratuito no Sistema Único de Saúde (SUS) em relação ao gasto total com saúde aumentou de 5,4\% em 2002 para 10,7\% em 2007 (6).

Uma das políticas do governo brasileiro, implantada em 1999, que visa a diminuir o gasto com saúde é o estímulo ao uso e à produção de medicamentos genéricos (7). No Brasil, os medicamentos industrializados são classificados em três grupos: referência, genérico e similar. O medicamento referência é o produto inovador registrado no órgão federal. Os genéricos são os medicamentos que se mostram intercambiáveis com o de referência por testes de equivalência farmacêutica e bioequivalência. Os demais são denominados similares e devem ser identificados por nome comercial (7).

O SUS fornece gratuitamente, para a população, uma relação de medicamentos essenciais (RENAME). Entretanto, cada município tem autonomia para definir sua própria lista de medicamentos, baseada na RENAME, mas sem incluir necessariamente todos os medicamentos da lista nacional. Portanto, os medicamentos da RENAME podem não estar disponíveis nos serviços públicos municipais. Nesses casos, os indivíduos precisam às vezes recorrer ao setor privado como única alternativa, ocasionando gastos não previstos, que passam a consumir grande parte do orçamento familiar (8). Nesse contexto, o gasto com o tratamento de doenças crônicas compromete uma proporção importante da renda dos trabalhadores assalariados (9).

O objetivo deste estudo foi avaliar a disponibilidade de medicamentos no setor público para tratar sete doenças crônicas relevantes em termos de saúde pública (hipertensão arterial sistêmica, diabetes, asma, depressão, dislipidemia, epilepsia e úlcera péptica) e a capacidade aquisitiva em dias do salário de um trabalhador com rendimento de 1 salário mínimo nacional de pagar esses tratamentos no setor privado.

\section{MÉTODOS}

Utilizou-se metodologia preconizada pela Organização Mundial da Saúde
(OMS) e Health Action International (HAI) (10) para a coleta padronizada de informações sobre preços, disponibilidade e capacidade de pagamento dos medicamentos. Essa metodologia permite comparações com dados já existentes e entre diferentes municípios e países.

O delineamento do estudo foi transversal, realizado em amostra de conveniência que incluiu seis municípios do Estado do Rio Grande do Sul: a capital, Porto Alegre (1,4 milhão de habitantes); dois municípios com menor índice de desenvolvimento humano (IDH), na região sul do Estado (Pelotas e Bagé); um município localizado na região mais desenvolvida (Caxias do Sul); um município na região metropolitana da capital (São Leopoldo); e um município na região central do estado (Santa Cruz do Sul). A população total desses seis municípios representa um quarto da população total do Estado.

Em cada município foram selecionadas, aleatoriamente, quatro unidades do setor público que dispensam medicamentos da atenção básica, o principal hospital de atendimento pelo SUS e cinco farmácias privadas, eleitas pela proximidade em relação às unidades públicas de saúde. Nos municípios onde a dispensação era realizada em unidades de saúde específicas, as mesmas foram incluídas e, posteriormente, selecionadas as demais unidades públicas de saúde. No Município de São Leopoldo, apenas dois estabelecimentos públicos foram incluídos, pois eram responsáveis por toda a distribuição de medicamentos do setor público na cidade. A tabela 1 apresenta o total de estabelecimentos de saúde capacitados a dispensar medicamentos e

TABELA 1. Total de estabelecimentos de saúde que dispensam medicamentos e total de estabelecimentos pesquisados no setor público e privado nos municípios estudados, Rio Grande do Sul, Brasil, 2008 e 2009

\begin{tabular}{|c|c|c|c|c|c|c|c|c|c|c|c|c|}
\hline \multirow{2}{*}{$\begin{array}{l}\text { Estabelecimentos } \\
\text { de saúde avaliados }\end{array}$} & \multicolumn{2}{|c|}{$\begin{array}{l}\text { Porto } \\
\text { Alegre }\end{array}$} & \multicolumn{2}{|c|}{$\begin{array}{l}\text { Santa Cruz } \\
\text { do Sul }\end{array}$} & \multicolumn{2}{|c|}{$\begin{array}{l}\text { Caxias } \\
\text { do Sul }\end{array}$} & \multicolumn{2}{|c|}{$\begin{array}{c}\text { São } \\
\text { Leopoldo }\end{array}$} & \multicolumn{2}{|c|}{ Bagé } & \multicolumn{2}{|c|}{ Pelotas } \\
\hline & $\mathrm{N}^{\mathrm{a}}$ & $n^{b}$ & $\mathrm{~N}$ & $\mathrm{n}$ & $\mathrm{N}$ & $\mathrm{n}$ & $\mathrm{N}$ & $\mathrm{n}$ & $\mathrm{N}$ & $\mathrm{n}$ & $\mathrm{N}$ & $\mathrm{n}$ \\
\hline \multicolumn{13}{|l|}{ Setor público } \\
\hline$U_{B S}{ }^{c}$ e $E S F^{d}$ & 118 & 3 & 21 & 3 & 39 & 3 & 0 & 0 & 19 & 2 & 35 & 2 \\
\hline Farmácia municipal & 0 & 0 & 1 & 1 & 0 & 0 & 1 & 1 & 1 & 1 & 1 & 1 \\
\hline Farmácia distrital ou ce & & & & & & & & & & & & \\
\hline de especialidades & 13 & 1 & 1 & 0 & 1 & 1 & 1 & 1 & 4 & 1 & 6 & 1 \\
\hline \multicolumn{13}{|l|}{ Setor privado } \\
\hline Farmácia privada & 720 & 5 & 75 & 5 & 155 & 5 & 85 & 5 & 55 & 5 & 191 & 5 \\
\hline
\end{tabular}

o total de estabelecimentos pesquisados no setor público e privado nos municípios estudados.

Os dados sobre a disponibilidade dos medicamentos estudados nos hospitais não foram incluídos, pois se referem ao fornecimento de medicamentos apenas para pacientes internados. No processo de seleção das farmácias privadas, não foram incluídas farmácias da mesma rede no mesmo município nem em outros municípios incluídos no estudo.

A coleta de dados foi realizada de novembro de 2008 a janeiro de 2009. Neste estudo foram utilizadas informações de 21 medicamentos, usados no tratamento de sete doenças crônicas. $\mathrm{Na}$ tabela 2 descreve-se a lista de comparação à qual cada medicamento pertence. A classificação dos medicamentos em listas é preconizada pela metodologia utilizada, visando possíveis comparações com outros países. Para cada medicamento foram pesquisados, nas farmácias privadas, os preços de venda dos medicamentos referência, genérico e similar de menor preço. No setor público brasileiro não ocorre venda de medicamentos, sendo coletadas apenas informações sobre disponibilidade dos três grupos de medicamentos.

A disponibilidade foi definida como presença de, pelo menos, uma unidade comercial visível do medicamento no momento da coleta dos dados. O cálculo da disponibilidade foi realizado para cada cidade, dividindo-se o número de estabelecimentos onde o medicamento foi encontrado pelo número de estabelecimentos onde ele deveria estar disponível, considerando se este era genérico, referência ou similar. Os resultados foram 
TABELA 2. Medicamentos estudados, posologia utilizada no cálculo da capacidade de pagamento e lista de comparação à qual o medicamento pertence, Rio Grande do Sul, Brasil, 2008 e 2009

\begin{tabular}{|c|c|c|c|c|}
\hline Doença & Medicamento & Apresentação ${ }^{a}$ & $\begin{array}{l}\text { Posologia } \\
\text { para } 30 \text { dias }^{a}\end{array}$ & $\begin{array}{l}\text { Lista de } \\
\text { comparação }\end{array}$ \\
\hline Asma & $\begin{array}{l}\text { Salbutamol inalatório } \\
\text { Beclometasona inalatório }\end{array}$ & $\begin{array}{l}100 \mathrm{mcg} / \text { dose } \\
250 \mathrm{mcg} / \text { dose }\end{array}$ & $\begin{array}{l}200 \text { doses }^{b} \\
200 \text { doses }^{c}\end{array}$ & $\begin{array}{l}\text { Global } \\
\text { Regional }\end{array}$ \\
\hline Depressão & $\begin{array}{l}\text { Amitriptilina } \\
\text { Fluoxetina }\end{array}$ & $\begin{array}{l}25 \mathrm{mg} / \mathrm{cp} \\
20 \mathrm{mg} / \mathrm{cáps}\end{array}$ & $\begin{array}{l}3 \mathrm{cp} / \mathrm{dia}^{\mathrm{b}} \\
1 \text { cáps/diac }\end{array}$ & $\begin{array}{l}\text { Global } \\
\text { Regional }\end{array}$ \\
\hline Diabetes & $\begin{array}{l}\text { Glibenclamida } \\
\text { Metformina }\end{array}$ & $\begin{array}{c}5 \mathrm{mg} / \mathrm{cp} \\
850 \mathrm{mg} / \mathrm{cp}\end{array}$ & $\begin{array}{l}2 \mathrm{cp} / \mathrm{dia}^{\mathrm{b}} \\
1 \mathrm{cp} / \mathrm{dia}^{\mathrm{c}}\end{array}$ & $\begin{array}{l}\text { Global } \\
\text { Regional }\end{array}$ \\
\hline Dislipidemia & $\begin{array}{l}\text { Sinvastatina } \\
\text { Atorvastatina }\end{array}$ & $\begin{array}{l}20 \mathrm{mg} / \mathrm{cp} \\
10 \mathrm{mg} / \mathrm{cp}\end{array}$ & $\begin{array}{l}1 \mathrm{cp} / \mathrm{dia}^{\mathrm{b}} \\
1 \mathrm{cp} / \mathrm{dia}^{\mathrm{d}}\end{array}$ & $\begin{array}{l}\text { Global } \\
\text { Regional }\end{array}$ \\
\hline Epilepsia & $\begin{array}{l}\text { Carbamazepina } \\
\text { Fenitoína }\end{array}$ & $\begin{array}{l}200 \mathrm{mg} / \mathrm{cp} \\
100 \mathrm{mg} / \mathrm{cp}\end{array}$ & $\begin{array}{l}2 \mathrm{cp} / \mathrm{dia}^{\mathrm{c}} \\
3 \mathrm{cp} / \mathrm{dia}^{\mathrm{c}}\end{array}$ & $\begin{array}{l}\text { Complementar } \\
\text { Regional }\end{array}$ \\
\hline $\begin{array}{l}\text { Hipertensão arterial } \\
\text { sistêmica }\end{array}$ & $\begin{array}{l}\text { Anlodipino } \\
\text { Atenolol } \\
\text { Enalapril } \\
\text { Captopril } \\
\text { Furosemida } \\
\text { Hidroclorotiazida } \\
\text { Metildopa } \\
\text { Propanolol } \\
\text { Verapamil }\end{array}$ & $\begin{array}{c}5 \mathrm{mg} / \mathrm{cp} \\
50 \mathrm{mg} / \mathrm{cp} \\
10 \mathrm{mg} / \mathrm{cp} \\
25 \mathrm{mg} / \mathrm{cp} \\
40 \mathrm{mg} / \mathrm{cp} \\
25 \mathrm{mg} / \mathrm{cp} \\
250 \mathrm{mg} / \mathrm{cp} \\
40 \mathrm{mg} / \mathrm{cp} \\
80 \mathrm{mg} / \mathrm{cp}\end{array}$ & $\begin{array}{l}1 \mathrm{cp} / \mathrm{dia}^{c} \\
1 \mathrm{cp} / \mathrm{dia}^{\mathrm{b}} \\
1 \mathrm{cp} / \mathrm{dia}^{\mathrm{c}} \\
2 \mathrm{cp} / \mathrm{dia}^{\mathrm{b}} \\
1 \mathrm{cp} / \mathrm{dia}^{\mathrm{c}} \\
1 \mathrm{cp} / \mathrm{dia}^{\mathrm{c}} \\
2 \mathrm{cp} / \mathrm{dia}^{\mathrm{c}} \\
3 \mathrm{cp} / \mathrm{dia}^{\mathrm{c}} \\
2 \mathrm{cp} / \mathrm{dia}^{\mathrm{c}}\end{array}$ & $\begin{array}{c}\text { Regional } \\
\text { Global } \\
\text { Regional } \\
\text { Global } \\
\text { Regional } \\
\text { Regional } \\
\text { Complementar } \\
\text { Complementar } \\
\text { Complementar }\end{array}$ \\
\hline Úlcera péptica & $\begin{array}{l}\text { Omeprazol } \\
\text { Ranitidina }\end{array}$ & $\begin{array}{l}20 \mathrm{mg} / \mathrm{cáps} \\
150 \mathrm{mg} / \mathrm{cp}\end{array}$ & $\begin{array}{l}1 \mathrm{caps}^{\mathrm{d} \mathrm{dia}^{\mathrm{b}}} \\
2 \mathrm{cp} / \mathrm{dia}^{\mathrm{c}}\end{array}$ & $\begin{array}{l}\text { Global } \\
\text { Regional }\end{array}$ \\
\hline
\end{tabular}

a $\mathrm{cp}=$ comprimido; caps = cápsula.

b Dosagem definida segundo padrão da OMS (10).

c Dosagem definida segundo Formulário Terapêutico Nacional de 2010 (12).

d Dosagem definida segundo Micromedex (13).

expressos em \% do número total de estabelecimentos que deveriam disponibilizar o medicamento em cada município.

A capacidade aquisitiva dos medicamentos foi estimada como o número de dias de salário que um trabalhador com rendimento de 1 salário mínimo nacional necessita para adquirir, em uma farmácia privada, a quantidade necessária de medicamento. Como as doenças crônicas necessitam de tratamento contínuo, a capacidade de pagamento foi estimada para 30 dias de tratamento. Assim, a mediana do preço de venda ao consumidor da quantidade necessária do medicamento para 1 mês de tratamento foi dividida pelo valor diário do salário mínimo nacional (R\$ 12,73; US\$ 5,50) vigente no período da coleta de dados, resultando no número de dias de salário necessários para pagar o tratamento. $\mathrm{O}$ salário mínimo nacional mensal bruto no final de 2008 era de $\mathrm{R} \$$ 415,00 (US\$ 178,50) (11). Após dedução de $8 \%$ para as contribuições do Instituto Nacional da Seguridade Social, o valor ajustado foi de R\$381,80 (US\$ 165,00), ou R\$12,73 (US\$ 5,50) por dia.

Na tabela 2 estão apresentadas as sete doenças crônicas estudadas, os 21 medicamentos e a posologia utilizada no cálculo da capacidade de pagamento. Os seguintes critérios foram utilizados para a definição da posologia: 1) para medicamentos da lista global, adotou-se o padrão de dosagem sugerido pela OMS/ HAI (10); 2) para os demais medicamentos que constam no Formulário Terapêutico Nacional de 2010 (12), adotou-se a dosagem mínima de manutenção; 3) para atorvastatina, único medicamento estudado que não se inclui em nenhum dos critérios anteriores, adotou-se a dosagem descrita na literatura (13).

A entrada e análise dos dados foram realizadas em uma aplicação especial para o Microsoft Excel denominada "Caderno de trabalho OMS/HAI versão 5.0" (10). Devido às peculiaridades do mercado brasileiro, foram analisados três conjuntos de dados: referência vs. genérico, referência vs. similar e genérico vs. similar. A metodologia prevê a análise apenas dos medicamentos de referência e dos genéricos, realizando-se, assim, uma adaptação ao contexto brasileiro. Foi efetuada a dupla entrada dos dados, seguida de checagem dos possíveis erros de digitação e correção do banco.

Este estudo foi aprovado pelo Comitê de Ética em Pesquisa da Secretaria Municipal de Porto Alegre (processo 001.022903.08.9). Todos os locais investigados concordaram em participar do estudo e assinaram termo de consentimento livre e esclarecido.

\section{RESULTADOS}

A pesquisa incluiu 22 estabelecimentos públicos e 30 farmácias privadas. A tabela 3 mostra a disponibilidade média dos medicamentos usados para tratar doenças crônicas em cada município, que variou de 83,3\% (São Leopoldo) a 97,6\% (Caxias do Sul). Dentre os 21 medicamentos pesquisados, o número de medicamentos fornecidos pelos municípios variou de 10 a 15 .

Os medicamentos usados no tratamento da asma foram os menos disponíveis, sendo que, em dois municípios (Bagé e Pelotas), esses medicamentos não eram disponibilizados na atenção básica. Foi encontrada disponibilidade de $100 \%$ para medicamentos usados nos tratamentos da diabetes e depressão. Para a HAS, a disponibilidade variou de 80,0\% (Bagé e Pelotas) a 100,0\% (São Leopoldo). Na época da coleta de dados, os medicamentos para dislipidemia não integravam o componente básico da assistência farmacêutica, e assim não eram disponibilizados na atenção básica dos municípios estudados.

A tabela 4 descreve a disponibilidade dos medicamentos genéricos e similares no setor público. Não foram encontrados medicamentos de referência nos locais pesquisados. Dos nove medicamentos pesquisados, utilizados no manejo da HAS, dois não eram disponibilizados em nenhum município (anlodipino e atenolol). Para os demais medicamentos antihipertensivos, a disponibilidade média dos similares foi maior que $60,0 \%$, e dos genéricos, menor que $20,0 \%$. O enalapril foi o medicamento para tratamento da HAS com menor disponibilidade total $(60,0 \%)$.

A maior presença dos medicamentos similares em relação aos genéricos no setor público pode ser observada na tabela 4, que mostra uma disponibilidade dos genéricos de $20,8 \%$ em média, contra $84,3 \%$ dos similares.

Todos os medicamentos investigados para tratamento de diabetes e epilepsia eram disponibilizados pelos seis municípios. Os medicamentos utilizados no tratamento da depressão, diabetes, epilepsia e úlcera péptica apresentaram disponibilidade superior a 80,0\%. Em relação aos medicamentos utilizados no manejo da asma, a disponibilidade da be- 
TABELA 3. Disponibilidade percentual média dos medicamentos usados para tratar doenças crônicas na atenção básica do setor público estratificada por município, Rio Grande do Sul, Brasil, 2008 e 2009

\begin{tabular}{|c|c|c|c|c|c|c|c|c|c|c|c|c|}
\hline \multirow[b]{2}{*}{ Doença $^{a}$} & \multicolumn{2}{|c|}{ Porto Alegre } & \multicolumn{2}{|c|}{$\begin{array}{l}\text { Santa Cruz } \\
\text { do Sul }\end{array}$} & \multicolumn{2}{|c|}{$\begin{array}{l}\text { Caxias } \\
\text { do Sul }\end{array}$} & \multicolumn{2}{|c|}{$\begin{array}{c}\text { São } \\
\text { Leopoldo }\end{array}$} & \multicolumn{2}{|c|}{ Pelotas } & \multicolumn{2}{|c|}{ Bagé } \\
\hline & $\mathrm{n}^{\mathrm{b}}$ & Médiac \% & $\mathrm{n}$ & Média \% & $\mathrm{n}$ & Média \% & $\mathrm{n}$ & Média \% & $\mathrm{n}$ & Média \% & $\mathrm{n}$ & Média \% \\
\hline Asma $^{\mathrm{d}, \mathrm{e}}$ & 2 & 62,5 & 2 & 50,0 & 1 & 100,0 & 2 & 50,0 & - & - & - & - \\
\hline Depressão ${ }^{f}$ & 1 & 100,0 & 2 & 100,0 & 1 & 100,0 & 1 & 100,0 & 1 & 100,0 & 1 & 100,0 \\
\hline Diabetes & 2 & 100,0 & 2 & 100,0 & 2 & 100,0 & 2 & 100,0 & 2 & 100,0 & 2 & 100,0 \\
\hline Epilepsia $^{f}$ & 2 & 100,0 & 2 & 100,0 & 2 & 100,0 & 2 & 50,0 & 2 & 100,0 & 2 & 100,0 \\
\hline HAS $^{9}$ & 5 & 90,0 & 6 & 95,8 & 7 & 85,7 & 7 & 100,0 & 5 & 80,0 & 5 & 80,0 \\
\hline Úlcera pépticah & 1 & 50,0 & 1 & 100,0 & 1 & 100,0 & 1 & 100,0 & - & - & 1 & 100,0 \\
\hline Todas & 13 & 83,7 & 15 & 90,9 & 14 & 97,6 & 15 & 83,3 & 10 & 95,0 & 11 & 96,0 \\
\hline $\begin{array}{l}\text { a Os medicamento } \\
\text { b Número de medi } \\
\text { c Disponibilidade } \\
\text { d Medicamento pa } \\
\text { e Medicamentos p } \\
\text { f Medicamentos S } \\
\text { belecimentos em } \\
\text { g HAS = hipertens } \\
\text { h Medicamentos p }\end{array}$ & $\begin{array}{l}\text { dislip } \\
\text { tos pe } \\
\text { percer } \\
\text { ha disp } \\
\text { ma nã } \\
\text { ao co } \\
\text { Alegr } \\
\text { arial si }\end{array}$ & $\begin{array}{l}\text { idemia não er } \\
\text { squisados dis } \\
\text { tual. } \\
\text { ensado em ur } \\
\text { o disponibiliza } \\
\text { ntrole pela Pol } \\
\text { e. } \\
\text { stêmica. }\end{array}$ & $\begin{array}{l}\text { nico } \\
\text { na } \\
\text { a SV }\end{array}$ & $\begin{array}{l}\text { nibilizados } n \\
\text { dos na aten } \\
\text { stabelecime } \\
\text { tenção básic } \\
\text { S/MS } 344 / 19\end{array}$ & $\begin{array}{l}\text { públi } \\
\text { n Pe } \\
\text { dispe }\end{array}$ & $\begin{array}{l}\text { básica no } p \\
\text { ta pelo munic } \\
\text { co em Caxias } \\
\text { otas e Bagé. } \\
\text { nsados em u }\end{array}$ & odo d & coleta de d & & em cinco & & dois esta- \\
\hline
\end{tabular}

clometasona $250 \mathrm{mcg} /$ dose, encontrada em três municípios, foi de $30,0 \%$, e do salbutamol $100 \mathrm{mcg} /$ dose, encontrado em quatro municípios, foi de $75,0 \%$.

A figura 1 apresenta a capacidade aquisitiva para o tratamento da diabetes e da HAS. O medicamento para diabetes que exigiria o menor número de dias de salário para pagamento de um tratamento mensal foi a glibenclamida similar (0,6 dias). Entre os medicamentos utilizados para tratamento da HAS, o captopril apresentou a maior diferença de preço entre o medicamento referência e os demais (referência $=6$ dias, genérico $=1,9$ dia e similar = 1,3 dia). O diurético hidrocloro- tiazida, também utilizado no tratamento da HAS, foi o que consumiu o menor número de dias de salário (referência $=$ 0,4 dias, genérico $=0,3$ dias e similar $=0,2$ dias). A capacidade aquisitiva para o tratamento da asma, dislipidemia, depressão, epilepsia e úlcera péptica aparece na figura 2. Para todos os medicamentos

TABELA 4. Disponibilidade percentual média dos medicamentos em estudo usados para tratar doenças crônicas na atenção básica do setor público estratificada por tipo de medicamento (genérico ou similar), Rio Grande do Sul, Brasil, 2008 e 2009

\begin{tabular}{|c|c|c|c|c|c|c|c|}
\hline \multirow[b]{2}{*}{ Doença crônica } & \multirow[b]{2}{*}{ Medicamento/forma farmacêutica } & \multirow[b]{2}{*}{ RENAME } & \multirow{2}{*}{$\begin{array}{l}\text { Elenco de } \\
\text { referência }\end{array}$} & \multirow[b]{2}{*}{ REMUME $^{\mathrm{b}}$} & \multicolumn{3}{|c|}{ Disponibilidade setor público ${ }^{C}$} \\
\hline & & & & & $\%$ Genérico $^{d}$ & $\%$ Similar & $\%$ Total $^{\mathrm{e}}$ \\
\hline Asma & $\begin{array}{l}\text { Beclometasona inalatório } 250 \mathrm{mcg} / \mathrm{dose} \\
\text { Salbutamol inalatório } 100 \mathrm{mcg} / \mathrm{dose}\end{array}$ & $\begin{array}{l}\operatorname{Sim} \\
\operatorname{Sim}\end{array}$ & $\begin{array}{l}\text { Sim } \\
\text { Sim }\end{array}$ & $\begin{array}{l}3 \\
4\end{array}$ & - & $\begin{array}{l}30,0 \\
75,0\end{array}$ & $\begin{array}{l}30,0 \\
75,0\end{array}$ \\
\hline Depressão $^{f}$ & $\begin{array}{l}\text { Amitriptilina cp } 25 \text { mg } \\
\text { Fluoxetina cáps } 20 \text { mg }\end{array}$ & $\begin{array}{l}\operatorname{Sim} \\
\operatorname{Sim}\end{array}$ & $\begin{array}{l}\text { Sim } \\
\text { Não }\end{array}$ & $\begin{array}{l}6 \\
1\end{array}$ & $\begin{array}{c}16,7 \\
0\end{array}$ & $\begin{array}{r}83,3 \\
100,0\end{array}$ & $\begin{array}{l}100,0 \\
100,0\end{array}$ \\
\hline Diabetes & $\begin{array}{l}\text { Glibenclamida cp } 5 \mathrm{mg} \\
\text { Metformina cp } 850 \mathrm{mg}\end{array}$ & $\begin{array}{l}\text { Sim } \\
\text { Sim }\end{array}$ & $\begin{array}{l}\operatorname{Sim} \\
\operatorname{Sim}\end{array}$ & $\begin{array}{l}6 \\
6\end{array}$ & $\begin{array}{c}0 \\
22,7\end{array}$ & $\begin{array}{r}100,0 \\
77,3\end{array}$ & $\begin{array}{l}100,0 \\
100,0\end{array}$ \\
\hline Dislipidemia & $\begin{array}{l}\text { Atorvastatina cp } 10 \mathrm{mg} \\
\text { Sinvastatina } \mathrm{cp} 20 \mathrm{mg}\end{array}$ & $\begin{array}{l}\text { Não } \\
\text { Não }\end{array}$ & $\begin{array}{l}\text { Não } \\
\text { Não }\end{array}$ & $\begin{array}{l}0 \\
0\end{array}$ & $\overline{0}$ & $\begin{array}{l}0 \\
0\end{array}$ & $\begin{array}{l}0 \\
0\end{array}$ \\
\hline Epilepsia $^{f}$ & $\begin{array}{l}\text { Carbamazepina cp } 200 \text { mg } \\
\text { Fenitoína cp } 100 \text { m }\end{array}$ & $\begin{array}{l}\text { Sim } \\
\text { Sim }\end{array}$ & $\begin{array}{l}\text { Sim } \\
\text { Sim }\end{array}$ & $\begin{array}{l}6 \\
6\end{array}$ & $\begin{array}{l}16,7 \\
50,0\end{array}$ & $\begin{array}{l}83,3 \\
41,7\end{array}$ & $\begin{array}{r}100,0 \\
83,3\end{array}$ \\
\hline $\begin{array}{l}\text { Hipertensão arterial } \\
\text { sistêmica }\end{array}$ & $\begin{array}{c}\text { Anlodipino cp } 5 \mathrm{mg} \\
\text { Atenolol cp } 50 \mathrm{mg} \\
\text { Captopril cp } 25 \mathrm{mg} \\
\text { Enalapril cp } 10 \mathrm{mg} \\
\text { Furosemida cp } 40 \mathrm{mg} \\
\text { Hidroclorotiazida cp } 25 \mathrm{mg} \\
\text { Metildopa cp } 250 \mathrm{~m} \\
\text { Propranolol cp } 40 \mathrm{mg} \\
\text { Verapamil cp } 80 \mathrm{mg}\end{array}$ & $\begin{array}{l}\text { Sim } \\
\text { Sim } \\
\text { Sim } \\
\text { Sim } \\
\text { Sim } \\
\text { Sim } \\
\text { Sim } \\
\text { Sim } \\
\text { Sim }\end{array}$ & $\begin{array}{l}\text { Sim } \\
\text { Sim } \\
\text { Sim } \\
\text { Não } \\
\text { Sim } \\
\text { Sim } \\
\text { Sim } \\
\text { Sim } \\
\text { Sim }\end{array}$ & $\begin{array}{l}0 \\
0 \\
6 \\
3 \\
6 \\
6 \\
5 \\
6 \\
4\end{array}$ & $\begin{array}{c}0 \\
0 \\
18,2 \\
0 \\
0 \\
9,1 \\
0 \\
0 \\
12,5\end{array}$ & $\begin{array}{r}0 \\
0 \\
81,8 \\
60,0 \\
100,0 \\
95,5 \\
83,3 \\
100,0 \\
71,3\end{array}$ & $\begin{array}{c}0 \\
0 \\
100,0 \\
60,0 \\
100,0 \\
100,0 \\
83,3 \\
100,0 \\
71,4\end{array}$ \\
\hline Úlcera péptica & $\begin{array}{l}\text { Ranitidina cp } 150 \text { mg } \\
\text { Omeprazol cáps } 20 \text { mg }\end{array}$ & $\begin{array}{l}\text { Sim } \\
\text { Sim }\end{array}$ & $\begin{array}{l}\text { Sim } \\
\text { Não }\end{array}$ & $\begin{array}{l}1 \\
4\end{array}$ & $\begin{array}{l}0 \\
0\end{array}$ & $\begin{array}{r}100,0 \\
82,5\end{array}$ & $\begin{array}{r}100,0 \\
82,5\end{array}$ \\
\hline
\end{tabular}

a Medicamentos do Elenco de Referência do Componente Básico da Assistência Farmacêutica, conforme portaria GM 3237 de 24 de dezembro de 2007 do Ministério da Saúde.

b REMUME = Relação Municipal de Medicamentos Essenciais. Número de cidades em que o medicamento está pactuado na REMUME ou algum outro programa de fornecimento do governo.

c No setor público não foi encontrado nenhum medicamento de referência, ou seja, o setor público só dispensou genéricos ou similares.

d Os medicamentos beclometasona, salbutamol e atorvastatina não possuem versão genérica.

e Disponibilidade total independentemente da categoria disponível.

† Medicamentos sujeitos a controle pela Portaria SVS/MS 344/1998 dispensados em um único estabelecimento público em cinco cidades e em dois estabelecimentos em Porto Alegre. 
FIGURA 1. Capacidade aquisitiva para tratamento de diabetes e hipertensão arterial sistêmica, Rio Grande do Sul, Brasil, 2008 e 2009

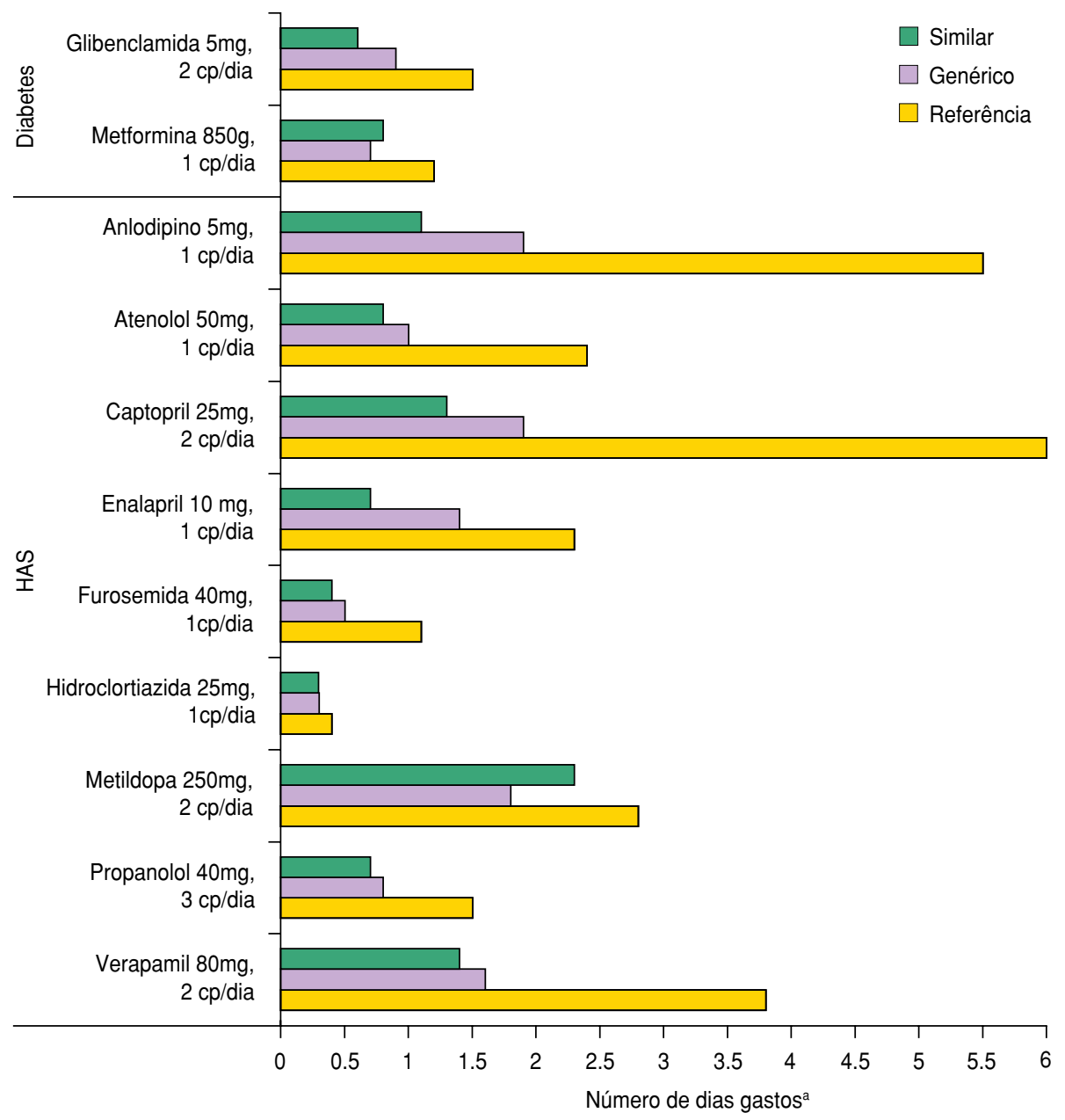

a Número de dias de salário necessários para pagar cada tipo de medicamento no setor privado, considerando-se um trabalhador com rendimento de 1 salário mínimo nacional.

pesquisados, ocorreu variação da capacidade aquisitiva de medicamentos genéricos, de referência e similares. A fluoxetina demonstra bem essa variação, exigindo 10,5 dias de salário para os medicamentos de referência, 2,8 dias para os genéricos e 1,8 dia para os similares. No tratamento da úlcera péptica, o omeprazol consumiu menos dias de salário quando comparado com a ranitidina. Para o tratamento da dislipidemia, a opção que consumiu menos dias de salário foi a sinvastatina similar (1,3 dia), enquanto que a atorvastatina similar requereu 8,4 dias.

\section{DISCUSSÃO}

De acordo com a orientação da OMS (14), a disponibilidade dos medicamentos essenciais deve ser superior a $80 \%$ para aumentar o acesso aos medicamen- tos. No presente estudo, o percentual médio de disponibilidade, considerando todos os medicamentos, ficou acima desse limite, embora tenha sido menor em alguns casos específicos, como o da asma e da úlcera péptica, em alguns municípios. Em todo caso, o percentual médio da disponibilidade dos tratamentos para doenças crônicas nos municípios pesquisados foi superior aos $74 \%$ descritos em um inquérito realizado em 2004 em cinco estados brasileiros (9). Isso também foi observado para medicamentos específicos, como no caso da metformina (genérico $=22 \%$ e similar $=$ $77,3 \%$ ), cuja disponibilidade foi superior à descrita por Pinto et al. em estudo desenvolvido em 30 municípios brasileiros em 2007 (15) (genérico $=3,3 \%$ e similar $=20 \%$ ). No entanto, essas diferenças podem não representar a realidade atual desses municípios (investigados em 2004 e 2007), onde a disponibilidade pode ter aumentado. Nos últimos anos ocorreram mudanças importantes na política para financiamento da assistência farmacêutica, que podem ter levado a uma qualificação da estrutura organizacional da assistência farmacêutica dos municípios.

Os resultados encontrados no presente estudo estão de acordo com o preconizado pela OMS. Entretanto, embora esse dado pareça positivo, é importante ressaltar que o estudo incluiu medicamentos que são parte de uma lista de medicamentos essenciais para o tratamento de doenças crônicas prevalentes. Portanto, o esperado seria uma disponibilidade de $100 \%$, dado o caráter essencial desses medicamentos. Ressalta-se que os medicamentos não fornecidos pelo SUS podem levar os usuários a adquiri-los no setor privado ou a não cumprir os tratamentos prescritos por impossibilidade de pagar (16).

Para uma avaliação dos resultados obtidos, é importante refletir sobre o financiamento da assistência farmacêutica no SUS, cuja responsabilidade é das três esferas de gestão - União, estados e municípios. Como o levantamento de dados foi realizado em unidades básicas de saúde, os medicamentos disponíveis deveriam atender aos agravos de saúde tratados nessa instância. Nem todos os medicamentos da RENAME têm financiamento definido no SUS (17). Por meio de legislação específica (18), são aprovadas as normas de execução e financiamento da assistência farmacêutica na atenção básica em saúde. Essa normativa, vigente na época do estudo, estabelecia o elenco de referência dos medicamentos que poderiam ser adquiridos com os recursos financeiros destinados ao componente básico da assistência farmacêutica, bem como os valores mínimos que deveriam ser aplicados por número de habitantes/ano em cada uma das três esferas de gestão para aquisição desses medicamentos. Observou-se que, dos 21 medicamentos pesquisados, 14 possuíam financiamento garantido por legislação específica, e apenas nove eram integrantes da Relação Municipal de Medicamentos Essenciais (REMUME) de todos os municípios.

É importante notar que a presença do medicamento nesse elenco definido por legislação específica não significa que o município é obrigado a disponibilizar o medicamento, pois cada local 
FIGURA 2. Capacidade aquisitiva para tratamento de asma, dislipidemia, depressão, epilepsia e úlcera péptica, Rio Grande do Sul, Brasil, 2008 e 2009

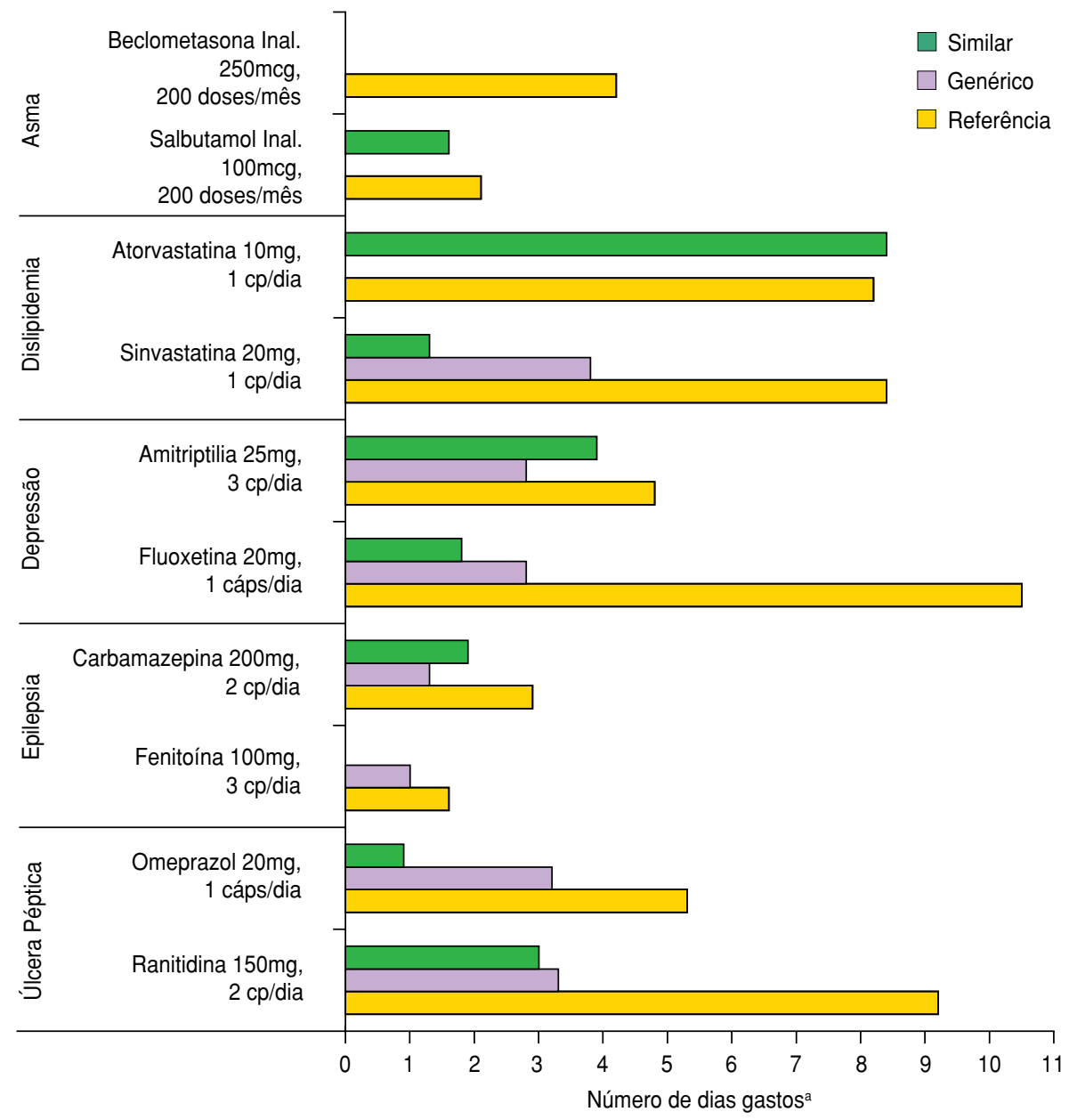

a Número de dias de salário necessários para pagar cada tipo de medicamento no setor privado, considerando-se um trabalhado com rendimento de 1 salário mínimo nacional.

tem autonomia para selecionar os medicamentos a serem fornecidos na atenção básica, desde que não haja prejuízo para o atendimento dos agravos prevalentes de acordo com o perfil epidemiológico da região. No entanto, ao considerar os medicamentos utilizados no manejo da asma (beclometasona e salbutamol), que possuem financiamento garantido, observa-se que esse preceito não foi respeitado. Como não existem, disponíveis no SUS, alternativas terapêuticas a esses agentes e, no momento da coleta de dados, beclometasona e salbutamol estavam disponibilizados em apenas três e quatro municípios, respectivamente, verificou-se que a população desses municípios não possuía garantia de tratamento na atenção básica para asma.

Entre os resultados do presente estudo, destacam-se a centralização da distribuição de alguns medicamentos e listas municipais de medicamentos bastante restritas, especialmente nos municípios mais pobres. Em São Leopoldo, a distribuição dos medicamentos é realizada em apenas dois locais. Além disso, em todos os municípios, os medicamentos sujeitos a controle especial também possuem distribuição centralizada em alguns locais. A prática de centralização, embora possa apresentar vantagens relacionadas a aspectos logísticos de armazenamento, controle de estoque e disponibilidade de recursos humanos, também contribui para dificultar o acesso, pois exige dos usuários gastos de tempo e dinheiro com deslocamento até os locais de distribuição. Em relação ao número de medicamentos disponibilizados pelos municípios, observa-se que os dois municípios mais pobres pesquisados (Bagé e Pelotas) são os que disponibilizam um menor número de medicamentos, sendo que, em Pelotas, para duas doenças, não existiam medicamentos disponíveis na rede de atenção básica. Isso representa falta de acesso e compromete a qualidade da assistência farmacêutica prestada. O processo de descentralização da política de saúde no Brasil, iniciado em 1999 (19), levou a um significativo incremento das atividades e da participação dos municípios na provisão de serviços de saúde. Assim, trouxe para os gestores a responsabilidade do ciclo da assistência farmacêutica. Porém, aparentemente, não são todos os estados e municípios que vêm executando essas atividades de forma satisfatória e compromissada, o que pode levar a falta de acesso aos medicamentos (20).

$\mathrm{O}$ não acesso gratuito aos medicamentos, principalmente os de uso contínuo, pode levar ao comprometimento da renda familiar ou favorecer o abandono do tratamento, o que pode ocasionar o agravamento do estado de saúde e a consequente expansão dos gastos com internações e atendimentos ambulatoriais (21). Além disso, a maioria da população atendida no serviço público de saúde é de baixa renda, sendo o fornecimento gratuito, normalmente, a única alternativa de acesso aos medicamentos (22). Os medicamentos anlodipino e atenolol não eram disponibilizados em nenhum município. Assim, os pacientes com prescrição desses medicamentos teriam que trabalhar de 1 a 5 dias exclusivamente para pagar o tratamento mensal em uma farmácia privada.

No momento da coleta de dados, os medicamentos utilizados no tratamento da dislipidemia, cuja responsabilidade de fornecimento era da esfera estadual, não estavam disponíveis nas unidades de atenção básica dos municípios. Para utilizá-los, seriam necessários de 1,3 a 8,4 dias de salário de pessoas que recebem 1 salário mínimo mensal, dependendo da marca disponível nas farmácias. Assim, esses medicamentos podem ter um forte impacto na renda. Atualmente, como resultado de mudanças na legislação (23), a sinvastatina passou a integrar o componente básico da assistência farmacêutica.

Os resultados indicam que a capacidade de pagamento, nos municípios estudados, pode estar prejudicando os tratamentos com medicamentos para doenças crônicas. Segundo Yang et al. (24), os tratamentos que consomem 1 
dia ou menos do salário do trabalhador são considerados como acessíveis para pagamento. Seguindo esse critério, no presente estudo, apenas os medicamentos similares do propranolol, furosemida, atenolol, enalapril, glibenclamida, omeprazol e todas as categorias da hidroclorotiazida eram acessíveis para pagamento. Resultado semelhante foi encontrado em estudo realizado em 30 municípios brasileiros, nos quais um trabalhador consumiria até 4 dias de trabalho para comprar o tratamento para hipertensão (captopril e hidroclorotiazida) no setor privado (15).

Os esquemas terapêuticos podem ser modificados conforme a gravidade da doença e o perfil do paciente. Para os medicamentos que não estão na lista global, optamos, neste estudo, por considerar o uso da dosagem mínima. Portanto, a capacidade de pagamento de alguns medicamentos pode estar subestimada. Por exemplo, para a metformina, a dosagem de manutenção para pacientes obesos é superior à dosagem utilizada no cálculo. Assim, o número de dias do salário para pagar o tratamento aumenta em $100 \%$ (genérico $=1,4$ dia, similar $=$ 1,5 dia e referência $=2,4$ dias). No caso da asma, cabe destacar que a quantidade de medicamento utilizada no cálculo de capacidade aquisitiva pode ser suficiente para mais de 1 mês de tratamento no caso dos pacientes com poucas crises asmáticas. Porém, para pacientes graves, essa quantidade pode ser insuficiente para 1 mês.

O salário diário de um trabalhador que recebe 1 salário mínimo nacional foi utilizado para estimar a capacidade aquisitiva. Porém, em muitos países, uma proporção substancial da população ganha menos do que esse valor (25). No Estado do Rio Grande do Sul, cerca de $20 \%$ da população vivem abaixo da linha de pobreza (US\$ 1,00/dia). Para um trabalhador com rendimento de até 1 salário mínimo nacional, o tratamento mensal com glibenclamida similar consome 0,6 dia do salário. Considerando que uma pessoa receba o valor de US\$ 1,00 por dia, o tratamento passa a consumir 3,1 dias do salário desse indivíduo, ou seja, torna-se inacessível. Nesse sentido, o desfecho mais provável, em vista da incapacidade de pagamento, é o subtratamento, ou mesmo seu abandono.

Apesar de os medicamentos similares possuírem maior disponibilidade no setor público, como já constatado por Miranda et al. em 2007 (26), além de menor custo do que os genéricos, a legislação (7) determina que apenas os medicamentos genéricos sejam considerados intercambiáveis com os de referência. No entanto, quando os medicamentos genéricos foram incorporados ao mercado farmacêutico brasileiro, já existia um mercado bem consolidado de similares que não estavam sujeitos aos mesmos testes de qualidade a que são submetidos os genéricos e que possuíam menores preços. Essa realidade ainda persiste, embora, desde 2003, uma mudança de legislação exija que medicamentos similares sejam submetidos aos mesmos testes exigidos para os genéricos no momento da renovação de registro. Contudo, os prazos para adequação a essa legislação não decorreram (27). Espera-se que a disponibilidade crescente dos medicamentos genéricos no mercado venha a contribuir para a capacidade de pagamento de tratamentos com qualidade assegurada (25).

Algumas limitações deste estudo devem ser consideradas. A metodologia prevê que a disponibilidade seja determinada através de uma lista específica de medicamentos sem alternativa de dosagem e opção terapêutica, coletada uma única vez em cada local. Assim, a disponibilidade de alguns tratamentos pode estar subestimada. Além disso, a metodologia considera disponível o medicamento que apresentar uma unidade posológica em estoque, sendo que seriam importantes informações sobre a existência de unidades suficientes para provisão do esquema terapêutico.

Ao extrapolar os resultados para o Brasil, devido ao perfil de morbidade de cada região e às diferentes prioridades na atenção à saúde, podem existir diferenças na disponibilidade. Entretanto, outros 45 estudos foram conduzidos em 36 países com a mesma metodologia, a qual é considerada uma fonte confiável de informações do setor farmacêutico dos países (15). Do mesmo modo, a metodologia permite a comparação de dados entre países com diferentes graus de organização e de desenvolvimento, sendo uma ferramenta de coleta sistemática e de análise padronizada, favorecendo a viabilidade financeira e replicação do estudo $(25,28)$.

Novos estudos com diferentes abordagens são necessários, visando a ampliar o conhecimento sobre a capacidade aquisitiva. Além de outras abordagens, é importante que os estudos que utilizaram a metodologia da OMS/HAI sejam repetidos rotineiramente como forma de acompanhar a evolução das políticas farmacêuticas do país pelo viés da metodologia OMS/HAI.

Para que o acesso aos medicamentos necessários para a população dos municípios brasileiros seja melhorado, é fundamental a constituição de equipes multidisciplinares locais, tecnicamente preparadas, para realizar a seleção dos medicamentos a serem disponibilizados em nível municipal, com base na RENAME e também nos preceitos da medicina baseada em evidências. Além disso, é importante que os gestores municipais conheçam a capacidade aquisitiva dos trabalhadores e o custo comparativo dos medicamentos, de forma a considerar o impacto na renda dos trabalhadores dos medicamentos não disponibilizados gratuitamente.

Agradecimentos. Este projeto foi financiando pelo Conselho Nacional de Desenvolvimento Científico e Tecnológico $(\mathrm{CNPq})$. Os autores agradecem a Martin Auton e Margaret Ewen (Health Action International) pelo apoio técnico na coleta, entrada e análise dos dados.

\section{REFERÊNCIAS}

1. Wong LLR, Carvalho JA. O rápido processo de envelhecimento populacional do Brasil: sérios desafios para as políticas públicas. Rev Bras Estud Popul. 2006;23(2):5-26.

2. Brasil, Ministério da Saúde. Assistência farmacêutica na atenção básica: instruções técnicas para sua organização. $2^{\mathrm{a}}$ ed. Brasília: Ministério da Saúde; 2006. Disponível em: http://portal.saude.gov.br/portal/arqui vos/pdf/assistencia_farmaceutica_na_aten cao_basica.pdf Acessado em 19 de janeiro de 2010.
3. Silveira FG, Osório RG, Piola SF. Os gastos das famílias com saúde. Cienc Saude Coletiva. 2002;7(4):719-31.

4. Menezes T, Campolina B, Silveira F, Servo L, Piola S. O gasto e a demanda das famílias em saúde: análise a partir da POF de 2002-2003. 
Em: Silveira FS, Servo LMS, Menezes T, Piola SF, eds. Gasto e consumo das famílias brasileiras contemporâneas. Brasília: IPEA; 2006. Pp. 313-44.

5. Barros A, Bertoldi A. Out-of-pocket health expenditure in a population covered by the Family Health Program in Brazil. Int J Epidemiol. 2008;37(4):758-65.

6. Vieira FS. Gasto do Ministério da Saúde com medicamentos: tendência dos programas de 2002 a 2007. Rev Saude Publica. 2009;43(4):674-81.

7. Brasil. Lei $9787 / 1999$. Disponível em: http:// www.planalto.gov.br/ccivil_03/leis/19787. htm Acessado em 19 de janeiro de 2012.

8. Diniz BPC, Servo LMS, Piola SF, Eirado M. Gasto das famílias com saúde no Brasil: evolução e debate sobre gasto catastrófico. Em: Silveira FS, Servo LMS, Menezes T, Piola SF, eds. Gasto e consumo das famílias brasileiras contemporâneas. Brasília: IPEA; 2006. Pp. 143-66.

9. Organização Pan-Americana da Saúde. Avaliação da assistência farmacêutica no Brasil: estrutura, processo e resultados. Brasília: OPAS/OMS/Ministério da Saúde; 2005. Disponível em: www.opas.org.br/medicamen tos/site/UploadArq/HSE_ASF_REM_1205. pdf Acessado em 4 de outubro de 2010.

10. World Health Organization/Health Action International. Medicine prices, availability, affordability and price components. Disponível em: www.haiweb.org/medicineprices Acessado em 4 de outubro de 2010

11. Brasil. Lei $11709 / 2008$. Disponível em: http:// www.portalbrasil.net/salariominimo_2008. htm Acessado em 10 de janeiro de 2012.

12. Brasil, Ministério da Saúde. Formulário terapêutico nacional 2010, Rename 2010. Disponível em: http://portal.saude.gov.br/ portal/arquivos/pdf/FTN_2010.pdf Acessado em 19 de janeiro de 2012.
13. Micromedex. Micromedex ${ }^{\circledR}$ (Healthcare Series). Disponível em: http://www.thomsonhc.com/ Acessado em 6 de outubro de 2009.

14. World Health Organization. Implementing the third WHO Medicines Strategy 2008-2013. Disponível em: http://apps.who.int/medi cinedocs/documents/s16821e/s16821e.pdf Acessado em 12 de janeiro de 2012.

15. Pinto CDBS, Miranda ES, Emmerick ICM, Costa NR, Castro CGSO. Preços e disponibilidade de medicamentos no Programa Farmácia Popular do Brasil. Rev Saude Publica. 2010;44(4):611-9.

16. Bertoldi AD, Helfer AP, Camargo AL, Tavares NUL, Kanavos P. Medicine prices, availability and affordability in southern Brazil: a study of public and private facilities. (The LSE Health Working Paper Series in Health Policy and Economics). Disponível em: http:// www2.lse.ac.uk/LSEHealthAndSocial Care/pdf/LSEHealthworkingpaperseries / LSEHWP18.pdf Acessado em 4 de outubro de 2010.

17. Vieira FS. Assistência farmacêutica no sistema público de saúde no Brasil. Rev Panam Salud Publica. 2010;27(2):149-56.

18. Brasil. Portaria 3 237/2007. Disponível em: http://portal.saude.gov.br/portal/arqui vos/pdf/Portaria_GM_N_3237.pdf Acessado em 10 de janeiro de 2012 .

19. Brasil. Portaria 176/1999. Disponível em: http://www.portaleducacao.com.br/farma cia/artigos/1133/portaria-n-176-de-8-demarco-de-1999 Acessado em 10 de janeiro de 2012.

20. Santos-Pinto CDB, Costa NR, Osorio-de-Castro CGS. Quem acessa o Programa Farmácia Popular do Brasil? Aspectos do fornecimento público de medicamentos. Cienc Saude Coletiva. 2011;16(6):2963-73.

21. Arrais PSD, Brito LL, Barreto ML, Coelho HLL. Prevalência e fatores determinantes do consumo de medicamentos no Município de Fortaleza, Ceará, Brasil. Cad Saude Publica. 2005;21(6):1737-46

22. Paniz VMV, Fassa AG, Facchini LA, Bertoldi $\mathrm{AD}$, Piccini RX, Tomasi E, et al. Acesso a medicamentos de uso contínuo em adultos e idosos nas regiões Sul e Nordeste do Brasil. Cad Saude Publica. 2008;24(2):267-80.

23. Brasil. Portaria 4 217/2010. Disponível em: http:/ / www.brasilsus.com.br/legislacoes/ gm/106972-4217.html Acessado em 10 de janeiro de 2012.

24. Yang H, Dib HH, Zhu M, Qi G, Zhang $X$. Prices, availability and affordability of essential medicines in rural areas of $\mathrm{Hu}$ bei Province, China. Health Policy Plan. 2010;25(3):219-29.

25. Cameron A, Ewen M, Ross-Degnan D, Ball D, Laing R. Medicine prices, availability, and affordability in 36 developing and middleincome countries: a secondary analysis. Lancet. 2009:373(9659):240-9.

26. Miranda ES, Pinto CDBS, Reis ALA, Emmerick ICM, Campos MR, Luiza VL, et al. Disponibilidade no setor público e preços no setor privado: um perfil de medicamentos genéricos em diferentes regiões do Brasil. Cad Saude Publica. 2009;25(10):2147-58.

27. Rumel D, Nishioka SA, Santos AAM. Intercambialidade de medicamentos: abordagem clínica e o ponto de vista do consumidor. Rev Saude Publica. 2006;40(5):921-7.

28. Madden JM, Meza E, Ewen M, Laing RO, Stephens P, Ross-Degnan D. Measuring medicine prices in Peru: validation of key aspects of WHO/HAI survey methodology. Rev Panam Salud Publica. 2010;27(4):291-9.

Manuscrito recebido em 21 de junho de 2011. Aceito em versão revisada em 7 de novembro de 2011.

ABSTRACT Objective. To assess the affordability by workers of drugs used for treatment of chronic diseases, as well as the availability of the reference, similar, or generic forms of these drugs in the public health care system.

\section{Affordability and availability of drugs for treatment of chronic diseases in the public health care system}

Methods. We employed the methodology recommended by the World Health Organization (WHO) and Health Action International (HAI) for the standardized collection of information on selling prices in the private sector and availability in the public health care system of drugs in six cities in the state of Rio Grande do Sul, Brazil. Data were collected from November 2008 to January 2009. Affordability was estimated as the number of salary days required for a worker receiving the national minimum wage to buy, in a private pharmacy, the amount of medication required for one month of treatment. Availability was assessed by the presence of these drugs in public health care system facilities. Results. Twenty-two public facilities and 30 private pharmacies were studied. Of 21 drugs used for the treatment of seven chronic disorders, only nine were available free of charge in the six cities. Mean availability ranged from $83.3 \%$ (São Leopoldo) to $97.6 \%$ (Caxias do Sul). Affordability ranged from 0.4 to 10.5 salary days for reference drugs, 0.2 to 8.4 salary days for similar drugs, and 0.3 to 3.8 salary days for generic drugs.

Conclusions. The overall availability of the drugs surveyed was higher than the $80 \%$ recommended by WHO. However, some treatments were not available, or had limited availability in the public system. Concerning affordability, the number of salary days required to buy these drugs may affect the continuation of drug treatments for chronic diseases.

Key words Economics, pharmaceutical; drugs, essential; drug price; chronic disease. 